\title{
Determination of Suitable Poly Bag Diameter for Microalgae Chlorella vulgaris Cultivation for Algal Biodiesel Production
}

\author{
Herath H.M.H.M., Bandara W.B.M.A.C.*, Rupasinghe C.P. \\ Department of Agricultural Engineering, Faculty of Agriculture, University of Ruhuna, \\ Sri Lanka \\ *chathu.anushk@gmail.com
}

\begin{abstract}
Consumption of fossil fuels is now widely accepted as an unsustainable solution to compensate the current energy demand. Fossil fuel is a depleting resource and its burning causes the accumulation of greenhouse gases in the atmosphere which has already exceeded the 'dangerously high' threshold of $450 \mathrm{ppm} \mathrm{CO} 2$-e. Hence, the solution is to find environmentally and economically sustainable energy production processes that are not only renewable, but also capable of sequestering atmospheric $\mathrm{CO}_{2}$. Among different alternatives, production of bio fuel from micro algae is considered as a sustainable solution which can address the above criteria successfully. Through photo-bioreactors systems allow for better control of the algae culture environment, but tend to be more expensive. Therefore this study was carried out to determine the suitable diameter for poly bag reactors used for microalgae Chlorella vulgaris with referring to the resulting dry matter yield and the oil content. Using Guillard \& Ryther's modified F medium, Chlorella vulgaris was grown in poly bag reactors with diameters of 5,10,15 and $30 \mathrm{~cm}$ under the same environmental condition for 14 days. Experimental design was Completely Randomise Design and the treatments were replicated three times. Algae were harvested by flocculation method using $\mathrm{NaOH}$ as a flocculation agent. Dry matter content (w/w) of each sample was measured and, the oil extraction was carried out by using Soxhlet method. Dry matter contents of Chlorella vulgaris in 5, 10, 15 and $30 \mathrm{~cm}$ diameter poly bag reactors were recorded as $0.0018,0.00116,0.0041$ and 0.00065 $\mathrm{gcm}^{-3}$ respectively. The oil contents of $5,10,15$ and $30 \mathrm{~cm}$ diameter poly bag cultures were $11.21 \%, 6.92 \% .17 .5 \%$ and $6.72 \%$ respectively. Higher Phosphate removal was observed in poly bag reactor with $15 \mathrm{~cm}$ diameter. The highest nitrate and phosphate contents were retained in $30 \mathrm{~cm}$ diameter poly bag reactor and recorded as 0.072 and $0.229 \mathrm{mg} / \mathrm{l}$ respectively. When the diameter exceeds $15 \mathrm{~cm}$, algae growing rate was decreased due to limited light penetration. Results revealed that $15 \mathrm{~cm}$ is the best diameter of poly bag reactors for growing Chlorella vulgaris, as the recorded dry matter and lipid contents of C. vulgaris related to the same was significantly high. In addition, it is an economically viable solution under local conditions.
\end{abstract}

Keywords: Biodiesel, Chlorella vulgaris, Dry matter content, Lipid content, Microalgae

Proceedings of the $22^{\text {nd }}$ International Forestry and Environment Symposium 2017 of the Department of Forestry and Environmental Science, University of Sri Jayewardenepura, Sri Lanka 